\title{
EFFECT OF SALTS UPON OXIDASE ACTIVITY OF APPLE BARK
}

\section{CONTRIBUTIONS FROM THE HULL BOTANICAL LABORATORY 263}

D. H. Rose, Henry R. KRAybili, and R. C. Rose

\section{(WITH FIVE FIGURES)}

\section{Introduction}

In an earlier paper (2I) one of the authors showed that there is a marked difference in the action of the salts of the alkali metals upon the fire-holding capacity of tobacco, even when the salts have similar anions. For instance, the carbonates of potassium, rubidium, and caesium promote the combustion of tobacco to a very much greater extent than the carbonates of sodium and lithium. The chlorides of sodium, lithium, and potassium retard the combustion, but the chloride of potassium is not nearly so effective as the chloride of sodium or lithium. In general, the salts of potassium, rubidium, and caesium are much more favorable to combustion than those of sodium and lithium.

It has been known for a long time that potassium is an essential element for the higher plants. Numerous attempts have been made to replace potassium by sodium, and, while apparently sodium can fulfil some of the functions of potassium, attempts to replace potassium entirely by sodium have been unsuccessful. The fact that potassium seems to have such a marked property of promoting the combustion of tobacco, and sodium does not, suggests that this particular property of potassium may have a relation to certain functions in the plant, which cannot be fulfilled by sodium. These facts suggested that a study of the effect of the alkali salts upon oxidase activity might be of interest. The work reported in this paper was done in I9I7. More extended studies were planned, but, since it has been impossible to carry them out completely at the present time, it seemed wise to report the results obtained.

Botanical Gazette, vol. 69] 


\section{Historical}

BERTRAND (5) was the first investigator to point out that the salts of metals influence oxidase activity. He showed that manganese salts greatly increase the oxidase activity of preparations from alfalfa. GESSARD (I5) found that the formation of melanin from tyrosin is increased in the presence of salts of the metals. BACH (4) substantiated GESSARD's results, and showed that aluminum sulphate, salts of calcium, magnesium, manganese, and zinc increase melanin formation from tyrosin. The effect of the salts is to increase the further change of the oxidation product rather than to activate the taking up of oxygen. Aluminum salts hasten the formation of purpurogallin from the yellow oxidation product of the action of oxidase upon pyrogallol. $\mathrm{BACH}$ believed that the oxidation process is retarded by the accumulation of the primary oxidation products, and that the salts act to release them. WoLFF (32) found that the oxidation of tyrosin by tyrosinase from Russula delica is increased by the addition of small quantities of disodiumphosphate. Porodko (26), Aso (3), Alsberg (2), and Ewart (II) have shown that salts of the metals give a blue color with guaiacum. Porodko and Ewart believed these salts to be inorganic oxidases. Porodko pointed out that those metals which form salts of two degrees of oxidation are particularly active. Alsberg, and also Ewart, confirmed Porodko's observation and found that the chlorides of many of the metals give a blue color with guaiacum. Alsberg attributed an important part in the reaction to the chlorine. EwART further found that the chlorides, nitrates, and sulphates of the same metal are not necessarily equally powerful in their action. Apparently the chlorides are more active than the sulphates. Various salts were found to act as sensitizers or retardants to oxidase activity. Potassium chloride, potassium iodide, potassium bromide, and potassium fluoride retard or even prevent the browning of pounded apple pulp.

Numerous investigators have shown that oxidase activity is affected by changes in reaction of the medium. BERTRAND (6) showed that the action upon guaiacol of laccase from Rhus succedanea is inhibited by $0.002 \mathrm{M}$ concentration of sulphuric acid. 
WolfF found tyrosinase from Russula delica most active in a solution neutral to phenolphthalein, and ABDERHALDEN and GugGENHEIM (I) found that tyrosinase is destroyed by $0.0 \mathrm{o} 6 \mathrm{~N}$ hydrochloric acid, and greatly retarded by $0.016 \mathrm{~N}$ sodium hydroxide. Rose (28) showed that the decrease in oxidase activity, as observed in the Bunzell apparatus, is due to an increase in the hydrogen ion concentration of the medium. ReED (27) found oxidase activity in potatoes and apples inhibited even by low hydrogen ion concentrations; and likewise BUNZELL (9) found the action of oxidase retarded with increasing hydrogen ion concentrations.

\section{Methods}

All but one of the experiments described in this paper were made with portions of apple bark which had been dried at $35^{-}-40^{\circ} \mathrm{C}$. for $2-3$ hours, ground fine enough to pass through a 40-mesh wire sieve, and stored air dry in zinc-capped Mason jars. One experiment was made with solutions of precipitated oxidase separated from aqueous extracts of healthy bark and of diseased bark by the addition of about ro volumes of alcohol. In order to obtain the precipitated oxidase, $2 \mathrm{gm}$. of bark were allowed to stand in a beaker with ro cc. of water and 5 drops of toluol for i hour. The extract was then squeezed out through moist cheesecloth on coarse filter paper. The beaker was washed with five I cc. portions of water and the filter paper finally with two more. There was then added $50 \mathrm{cc}$. of 95 per cent alcohol to the filtrate (concentration of alcohol about 70 per cent) and the whole allowed to stand for Io minutes. The flocculent precipitate which had formed was collected on a hard filter by gentle suction with a filter pump. There was then added I50 cc. more alcohol to the filtrate (concentration of alcohol now about 90 per cent) and the whole allowed to stand for I hour, since precipitation was slow, before this second fraction was collected on the filter with the first. The precipitate was dissolved in water and used immediately, as described later.

The stock solutions of all of the salts tested were made to a concentration of $0.5 \mathrm{~N}$. Potassium chloride, manganese chloride, ferrous chloride, and ferric chloride were used also in the additional 
concentrations of $0 . \mathrm{I}$ and $0.0 \mathrm{IN}$. Since there was always $5 \mathrm{cc}$. of water in the apparatus, the final concentration of the salt, there was $0.1 \mathrm{~N}$ for $0.5 \mathrm{~N}$ solutions and 0.02 and $0.002 \mathrm{~N}$ for o. I $\mathrm{N}$ and $0.01 \mathrm{~N}$ solutions used.

Oxidation was measured in centimeters of mercury rise by means of the simplified Bunzell apparatus (8). The shaking machine was run at the rate of 106 complete excursions per minute. All experiments were run for 3 , hours, readings being taken every ${ }_{5} 5$ minutes, and a final reading the following morning. When bark was used, the mixtures in the apparatus contained O.I gm. of bark, I cc. of salt solution, and $4 \mathrm{cc}$. of I per cent pyrogallol solution or salt and pyrogallol with bark omitted, the second combination serving as a control on the first. Preliminary experiments had shown that during the time in which these experiments were run the autooxidation of the pyrogallol was usually not more than the equivalent of $0.15 \mathrm{~cm}$. mercury rise. In the experiment with precipitated oxidase, the precipitate from $2 \mathrm{gm}$. of bark was dissolved in $20 \mathrm{cc}$. of water, and $2 \mathrm{cc}$. of the solution, containing the dissolved precipitate obtained from $0.2 \mathrm{gm}$. of bark, were put in each apparatus, together with the usual amount of pyrogallol and water. All tests were run in duplicate. Two controls were run with each experiment, one containing only water, the other bark (or oxidase solution), pyrogallol, and water, but without the addition of salts.

The figures for $\mathrm{P}_{\mathrm{н}}$ given in table VII were obtained by means of the apparatus described by Rose (28).

\section{Discussion}

The chlorides in general retard oxidase activity. The chlorides of potassium, sodium, and lithium depress markedly the oxidation of pyrogallol by bark (table I). Similar results were obtained with all the other chlorides tested, except ferrous chloride (table VI). Ferrous chloride in o. I $\mathrm{N}$ concentration with bark and pyrogallol showed I. $79 \mathrm{~cm}$. mercury rise, and with pyrogallol alone $\mathrm{I} .45 \mathrm{~cm}$., compared with the control of pyrogallol and bark as $1.00 \mathrm{~cm}$. Since ferrous chloride is readily oxidized when exposed to the air, it is quite probable that the oxygen absorption for the most part represents that absorbed in the oxidation of ferrous chloride. 


\section{Results}

The results of the experiments are shown in tables I-VII and figs. I -5 .

\section{TABLE I}

EFfect of o. I N KCl, $\mathrm{NaCl}$, and LiCl on oxidation of pyrogallol by powdered HEALTHY APPLE BARK; TEMPERATURE $23 \cdot 2-23 \cdot 6^{\circ} \mathrm{C}{ }^{*}$

\begin{tabular}{|c|c|c|c|c|c|c|c|}
\hline \multirow{2}{*}{ TIME OF READING } & \multicolumn{3}{|c|}{ No BARK } & \multicolumn{4}{|c|}{ BARK } \\
\hline & $\mathrm{KCl}$ & $\mathrm{NaCl}$ & $\mathrm{LiCl}$ & Check & $\mathrm{KCl}$ & $\mathrm{NaCl}$ & $\mathrm{LiCl}$ \\
\hline \multicolumn{8}{|l|}{ May 2 I } \\
\hline I $2.30 \ldots$ & 0.00 & 0.00 & 0.00 & 0.00 & 0.00 & 0.00 & 0.00 \\
\hline I $2.45 \ldots \ldots$ & 0.03 & O.I3 & 0.00 & 0.03 & 0.06 & 0.07 & 0.03 \\
\hline I.০o..... & 0.03 & o. I 3 & 0.00 & 0.08 & 0.05 & O. I I & 0.05 \\
\hline I. I $5 \ldots \ldots$ & 0.08 & 0.20 & 0.00 & 0.23 & o. I 5 & $0:$ I8 & 0.15 \\
\hline I. $30 \ldots$. & 0.05 & O. I7 & 0.02 & 0.25 & O. I 5 & o. I8 & O.I 5 \\
\hline I $.45 \ldots \ldots$ & 0.05 & O.I3 & 0.00 & 0.33 & O. I 5 & $0.2 \mathrm{I}$ & O.I5 \\
\hline $2.00 \ldots \ldots$ & 0.07 & 0.18 & 0.00 & $0.3^{8}$ & 0.15 & 0.24 & 0.16 \\
\hline $2.15 \ldots \ldots$ & 0.08 & O. I9 & 0.05 & 0.43 & O. I9 & 0.27 & $0.2 \mathrm{I}$ \\
\hline $2.30 \ldots \ldots$ & 0.08 & O. I9 & 0.04 & 0.45 & o. I9 & $0.3 \mathrm{I}$ & 0.25 \\
\hline $2.45 \ldots \ldots$ & 0.07 & 0.17 & 0.05 & 0.45 & 0.25 & 0.30 & 0.25 \\
\hline $3.00 \ldots$ & 0.05 & o. I6 & 0.05 & 0.50 & 0.23 & 0.32 & 0.26 \\
\hline $3.15 \ldots$ & 0.09 & O.I9 & 0.06 & 0.65 & 0.26 & 0.36 & 0.29 \\
\hline May $2 \cdot 30 \ldots$. & 0.10 & 0.20 & 0.05 & 0.68 & 0.28 & 0.35 & 0.32 \\
\hline $8.40 \ldots$ & 0.00 & 0.00 & 0.00 & I. 25 & 0.80 & 0.74 & 0.77 \\
\hline
\end{tabular}
only water.

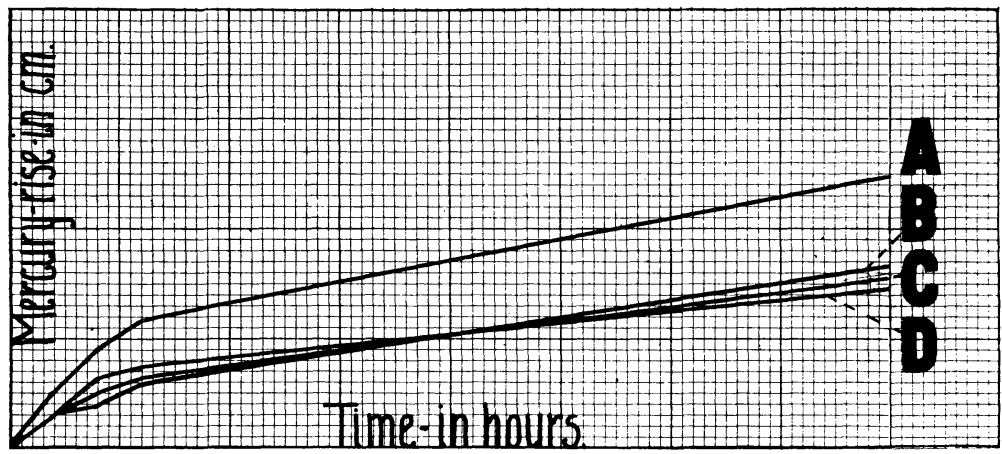

123

FIG. I.-Effect of $\mathrm{KCl}, \mathrm{NaCl}$, and $\mathrm{LiCl}$ on oxidation of pyrogallol by powdered healthy apple bark: A, control (bark and pyrogallol); $\mathrm{B}, \mathrm{KCl}+$ bark and pyrogallol; $\mathrm{C}, \mathrm{NaCl}+$ bark and pyrogallol; $\mathrm{D}, \mathrm{LiCl}+$ bark and pyrogallol. 


\section{TABLE II}

EFFECT OF O. IO N ALKALI CARBonates ON OXIDATION OF PYROGaLlol BY POWDERED HEALTHY APPLE BARK; TEMPERATURE $29 \cdot 3-30.0^{\circ} \mathrm{C}$.

\begin{tabular}{|c|c|c|c|c|c|c|c|}
\hline \multirow{2}{*}{ Time of REAding } & \multicolumn{3}{|c|}{ No BARK } & \multicolumn{4}{|c|}{ BARK } \\
\hline & $\mathrm{K}_{2} \mathrm{CO}_{3}$ & $\mathrm{Na}_{2} \mathrm{CO}_{3}$ & $\mathrm{Li}_{2} \mathrm{CO}_{3}$ & Check & $\mathrm{K}_{2} \mathrm{CO}_{3}$ & $\mathrm{Na}_{2} \mathrm{CO}_{3}$ & $\mathrm{Li}_{2} \mathrm{CO}_{3}$ \\
\hline \multicolumn{8}{|l|}{ June I3 } \\
\hline $1.30 \ldots \ldots$ & o. o & 0.00 & 0.00 & 0.00 & 0.00 & 0.00 & 0.00 \\
\hline I $.45 \ldots \ldots$ & 0.55 & 0.50 & 0.54 & 0.14 & 0.08 & 0.10 & 0.13 \\
\hline $2.00 \ldots \ldots$ & I. 35 & I. I3 & I. I9 & 0.19 & 0.48 & 0.47 & 0.48 \\
\hline $2.1_{5} \ldots \ldots$ & I. 75 & I. 55 & I. 64 & 0.24 & 0.75 & 0.85 & 0.82 \\
\hline $2.30 \ldots \ldots$ & 2. IO & I. 78 & I. 92 & 0.34 & 0.93 & 1.02 & I. 02 \\
\hline $2.45 \ldots \ldots$ & 2.40 & 2.05 & 2. 12 & 0.44 & I. I6 & I. 27 & I. 23 \\
\hline $3.00 \ldots \ldots$ & 2.50 & 2.15 & 2.24 & 0.49 & I. 33 & I. 42 & I. 33 \\
\hline $3.15 \ldots$ & 2.60 & 2.28 & 2.34 & 0.54 & I. 44 & I. $5^{2}$ & I. 50 \\
\hline $3.30 \ldots \ldots$ & 2.90 & $2.4 \mathrm{I}$ & 2.55 & 0.63 & 1.63 & I. 76 & I. 74 \\
\hline $3.45 \ldots \ldots$ & 3.00 & 2.47 & 2.57 & 0.68 & I. 79 & I. 86 & I. 85 \\
\hline $4.00 \ldots \ldots$ & 3.05 & 2.54 & 2.64 & 0.74 & 1.87 & I. 97 & I. 93 \\
\hline $4.15 \ldots$ & 3.08 & 2.60 & 2.69 & 0.79 & I. 93 & 2.02 & I. 99 \\
\hline $4.30 \ldots$ & 3.17 & 2.68 & 2.75 & 0.86 & 2.08 & 2.19 & 2.22 \\
\hline $\begin{array}{r}\text { June I } 4 \\
8.30 .\end{array}$ & & 2.63 & 2.74 & I. 29 & 2.70 & $2.6 \mathrm{I}$ & 2.85 \\
\hline 0.30 & 3.17 & & 2.74 & 1.29 & 2.70 & 2.01 & 2.05 \\
\hline
\end{tabular}

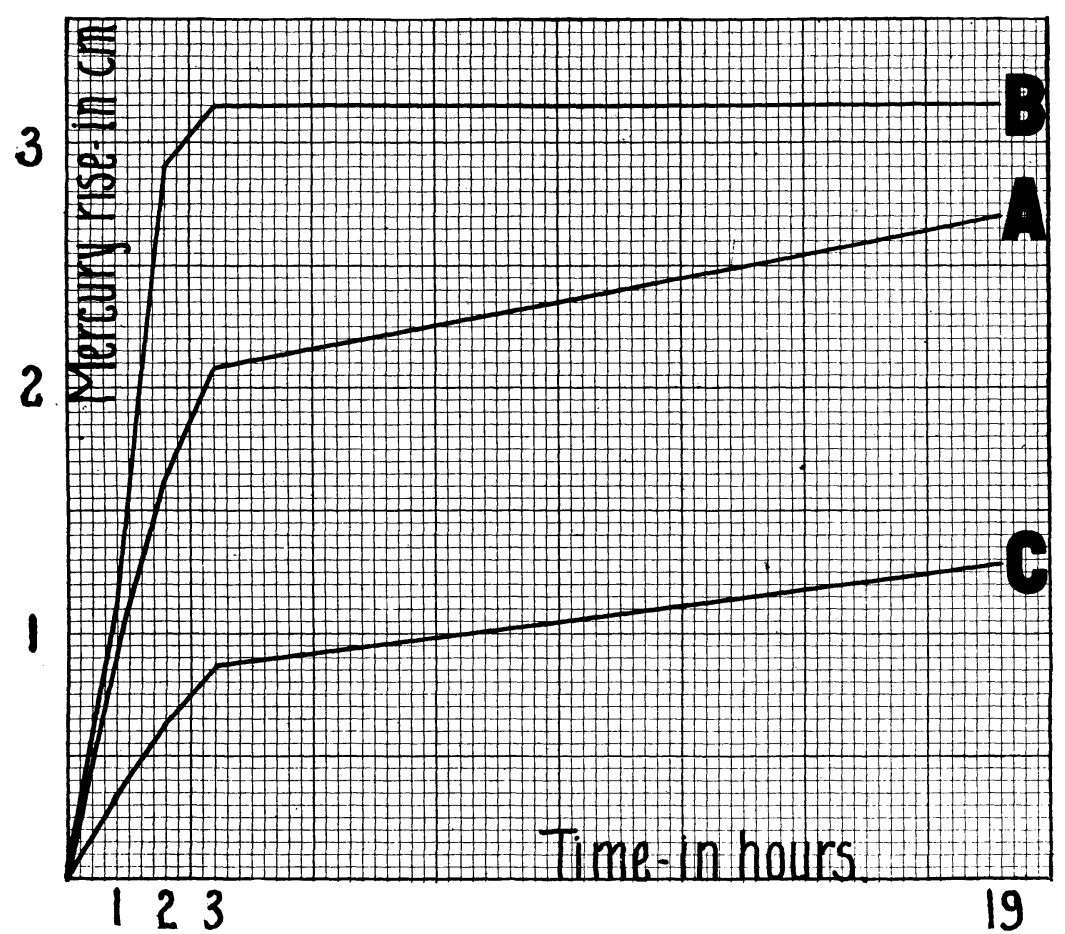

Fig. 2.-Effect of $\mathrm{K}_{2} \mathrm{CO}_{3}$ on oxidation of pyrogallol, with and without bark (healthy): $\mathrm{A}, \mathrm{K}_{2} \mathrm{CO}_{3}+$ bark and pyrogallol; $\mathrm{B}, \mathrm{K}_{2} \mathrm{CO}_{3}+$ pyrogallol; C, control (bark and pyrogallol) ontent downloaded from 129.105.215.146 on September 09, 2016 21:36:14 PM All use subject to University of Chicago Press Terms and Conditions (http://www.journals.uchicago.edu/t-and-c 
TABLE III

EFFeCT OF 0 . IO $\mathrm{N} \mathrm{KCl}$ AND $\mathrm{K}_{2} \mathrm{CO}_{3}$ ON OXIDATION OF PYROGALlOL BY POWDERED DISEASED APPLE BARK; TEMPERATURE $27.8-29.0^{\circ} \mathrm{C}$.

\begin{tabular}{|c|c|c|c|c|c|}
\hline \multirow{2}{*}{ Time of REAding } & \multicolumn{2}{|c|}{ No BARK } & \multicolumn{3}{|c|}{ BARK } \\
\hline & $\mathrm{K}_{2} \mathrm{CO}_{3}$ & $\mathrm{KCl}$ & Check & $\mathrm{K}_{2} \mathrm{CO}_{3}$ & $\mathrm{KCl}$ \\
\hline \multicolumn{6}{|l|}{ March Io } \\
\hline 10.00. & 0.00 & 0.00 & 0.00 & 0.00 & 0.00 \\
\hline $10.15 \ldots \ldots \ldots$ & 0.68 & -0.05 & 0.13 & 0.46 & 0.16 \\
\hline $10.30 \ldots \ldots \ldots$ & I. 24 & 0.00 & 0.30 & 0.90 & 0.33 \\
\hline Io. $45 \ldots \ldots \ldots$ & I. 65 & 0.00 & 0.50 & I. 25 & 0.38 \\
\hline II $.00 \ldots \ldots \ldots$ & I. 98 & -0.08 & 0.65 & I. 50 & 0.48 \\
\hline II I I $\ldots \ldots \ldots$ & 2.25 & -0.03 & 0.72 & I. 72 & 0.60 \\
\hline II $30 \ldots \ldots \ldots$ & $2 \cdot 38$ & -0.03 & 0.85 & I. 93 & 0.69 \\
\hline II $.45 \ldots \ldots \ldots$ & $2.5^{2}$ & 0.00 & 0.99 & 2.09 & 0.82 \\
\hline $12.00 \ldots \ldots \ldots$ & 2.65 & -0.05 & I. 04 & 2.22 & 0.88 \\
\hline $12.15 \ldots \ldots \ldots$ & 2.75 & 0.00 & I. I 5 & 2.35 & 0.95 \\
\hline $12.30 \ldots \ldots \ldots$ & 2.78 & -0.05 & I. I8 & 2.39 & 0.95 \\
\hline $\mathrm{I} 2.45 \ldots \ldots \ldots$ & 2.85 & -0.08 & I. 25 & 2.55 & I. .0 \\
\hline $\mathbf{I} .00 \ldots \ldots \ldots$ & 2.99 & -0.05 & I. 38 & 2.65 & I. IO \\
\hline \multicolumn{6}{|l|}{ March I I } \\
\hline $9.45 \ldots$ & $3 \cdot 53$ & -0.10 & 2.20 & $3 \cdot 73$ & I. 73 \\
\hline
\end{tabular}

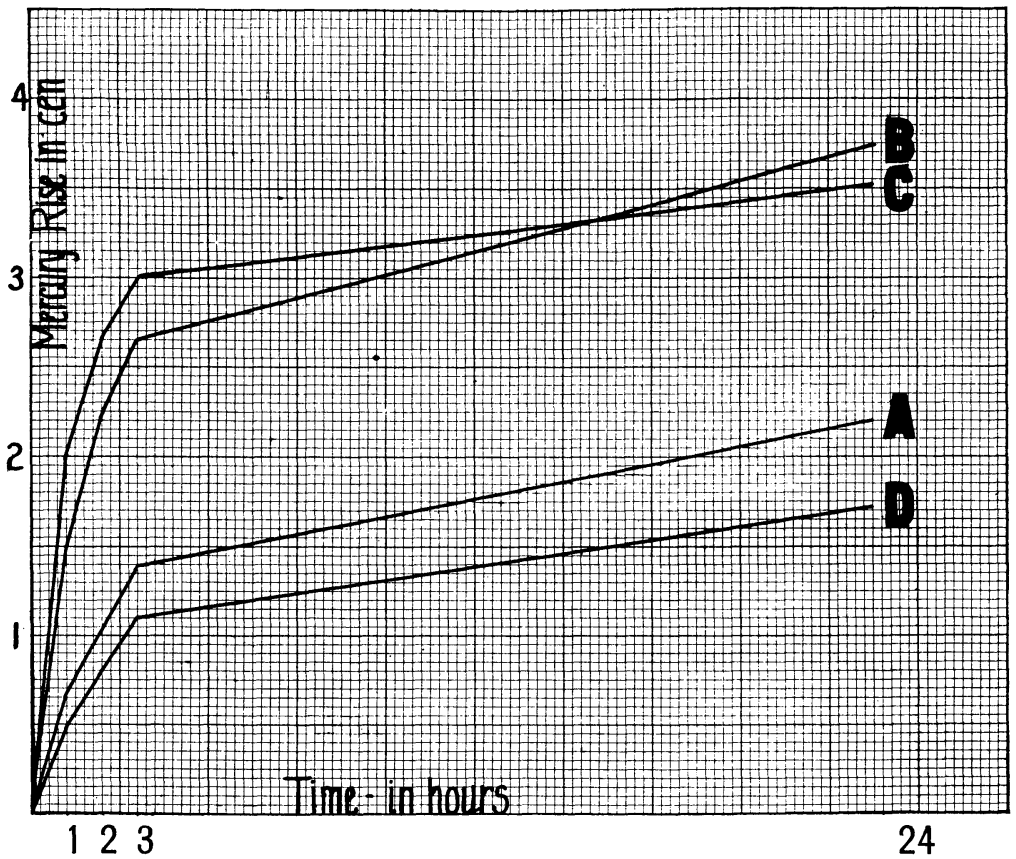

FIG. 3.-Effect of $\mathrm{KCl}$ and $\mathrm{K}_{2} \mathrm{CO}_{3}$ on oxidation of pyrogallol with and without bark (diseased): A, control (bark and pyrogallol); $\mathrm{B}, \mathrm{K}_{2} \mathrm{CO}_{3}+$ bark and pyrogallol; $\mathrm{C}, \mathrm{K}_{2} \mathrm{CO}_{3}$ +pyrogallol; $\mathrm{D}, \mathrm{KCl}+$ bark and pyrogallol $(\mathrm{KCl}+$ pyrogallol gave no oxidation).

This content downloaded from 129.105.215.146 on September 09, 2016 21:36:14 PM

All use subject to University of Chicago Press Terms and Conditions (http://www.journals.uchicago.edu/t-and-c 


\section{TABLE IV}

Effect of o. io $\mathrm{N}$ potassium tartrate, sodium oxalate, and $\mathrm{Ca}\left(\mathrm{NO}_{3}\right)_{2}$ on OXIDATION OF PYROGALLOL BY POWDERED HEALTHY APPLE BARK;

TEMPERATURE $29.2-30.2^{\circ} \mathrm{C}$.

\begin{tabular}{|c|c|c|c|c|c|c|c|}
\hline \multirow{2}{*}{ TiMe of REAding } & \multicolumn{3}{|c|}{ No BARK } & \multicolumn{4}{|c|}{ BARK } \\
\hline & $\begin{array}{c}\text { Potassium } \\
\text { tartrate }\end{array}$ & $\begin{array}{l}\text { Sodium } \\
\text { oxalate }\end{array}$ & $\mathrm{Ca}\left(\mathrm{NO}_{3}\right)_{2}$ & Check & $\begin{array}{c}\text { Potassium } \\
\text { tartrate }\end{array}$ & $\begin{array}{l}\text { Sodium } \\
\text { oxalate }\end{array}$ & $\mathrm{Ca}\left(\mathrm{NO}_{3}\right)_{2}$ \\
\hline \multicolumn{8}{|l|}{ June 22} \\
\hline I. $30 \ldots$ & 0.00 & 0.00 & 0.00 & 0.00 & 0.00 & 0.00 & 0.00 \\
\hline $\mathrm{I} .45 \ldots \ldots$ & O.II & 0.08 & 0.18 & 0.10 & 0.14 & 0.20 & 0.08 \\
\hline $2.00 \ldots$ & 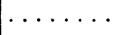 & $\ldots \ldots$ & $\ldots \ldots$ & 0.25 & 0.28 & 0.25 & 0.20 \\
\hline $2.15 \ldots \ldots$ & 0.25 & 0.10 & 0.20 & 0.30 & 0.36 & 0.35 & 0.23 \\
\hline 2.30. & and & $\ldots \ldots$ & $\ldots \ldots \ldots$ & 0.38 & 0.48 & 0.46 & 0.30 \\
\hline $2.45 \ldots$ & 0.35 & O.I9 & 0.20 & 0.43 & 0.58 & 0.55 & 0.35 \\
\hline $3.00 \ldots$ & $\ldots \ldots$ & $\ldots \ldots$ & $\ldots \ldots$ & 0.55 & 0.64 & 0.64 & 0.40 \\
\hline $3.15 \ldots$ & 0.48 & $0.3 \mathrm{I}$ & 0.28 & $0.5^{8}$ & 0.76 & 0.78 & 0.50 \\
\hline 3.30. & $\ldots \ldots$ & $\ldots \ldots \ldots$ & 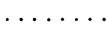 & 0.70 & 0.98 & 0.80 & 0.55 \\
\hline 3.45 & & & $\ldots \ldots$ & 0.73 & 0.95 & 0.96 & 0.59 \\
\hline 4.00. & 0.68 & $0.3^{8}$ & 0.35 & 0.80 & $\mathrm{I} .03$ & 1.00 & 0.60 \\
\hline $4.15 \ldots$ & $\ldots \ldots$ & & & 0.90 & I.I3 & I. IO & 0.73 \\
\hline 4.30. & \multicolumn{6}{|c|}{ June 23} & 0.70 \\
\hline $\begin{array}{r}\text { June } 23 \\
8.20\end{array}$ & 0.95 & 0.68 & 0.23 & I. 20 & I. 53 & I.6o & 0.98 \\
\hline & & & & & & & \\
\hline
\end{tabular}

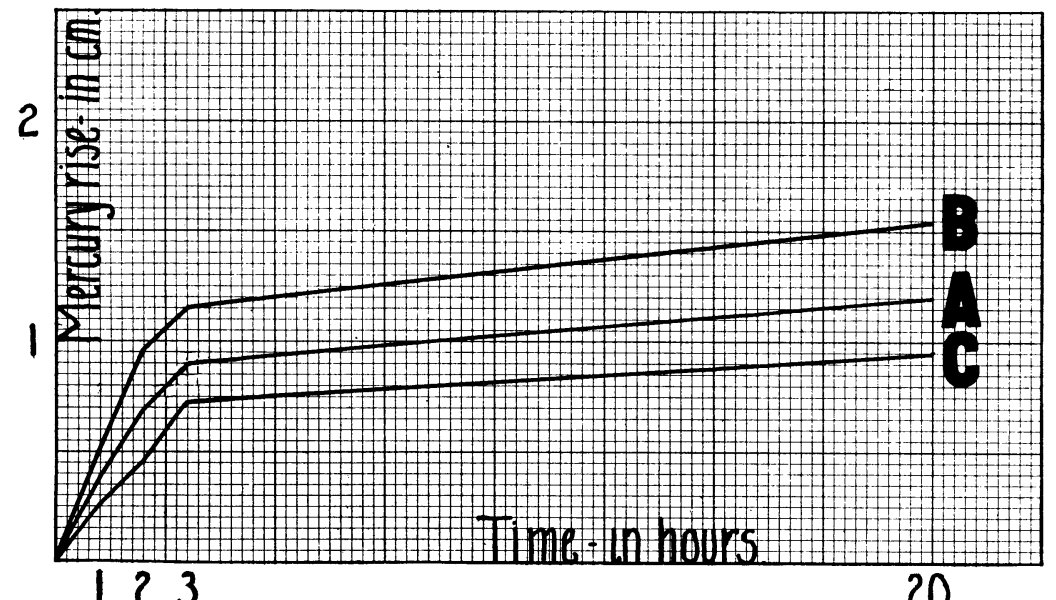

FIG. 4.-Effect of potassium tartrate on oxidation of pyrogallol with and without bark (healthy): A, control (bark and pyrogallol); B, potassium tartrate+bark and pyrogallol; C, potassium tartrate+pyrogallol.

This content downloaded from 129.105.215.146 on September 09, 2016 21:36:14 PM All use subject to University of Chicago Press Terms and Conditions (http://www.journals.uchicago.edu/t-and-c 


\section{TABLE V}

EFFEct of 0 . IO $\mathrm{N} \mathrm{MnCl}_{2}$ AND $\mathrm{K}_{2} \mathrm{SO}_{4}$ ON OXIDATION OF PYROgallol by PRECIPITATED OXIDASE FROM BOTH HEALTHY AND DISEASED APPLE BARK; TEMPERATURE $29 \cdot 5-30.2^{\circ} \mathrm{C}$.

\begin{tabular}{|c|c|c|c|c|c|c|}
\hline \multirow{2}{*}{ Time of READING } & \multicolumn{3}{|c|}{ Healthy } & \multicolumn{3}{|c|}{ DISEASED } \\
\hline & Check & $\mathrm{MnCl}_{2}$ & $\mathrm{~K}_{2} \mathrm{SO}_{4}$ & Check & $\mathrm{MnCl}_{2}$ & $\mathrm{~K}_{2} \mathrm{SO}_{4}$ \\
\hline June $2 \mathrm{I}$ & & & & & & \\
\hline$\quad \mathrm{I} .45 \ldots \ldots \ldots$ & 0.00 & 0.00 & 0.00 & 0.00 & 0.00 & 0.00 \\
\hline $2.00 \ldots \ldots \ldots \ldots$ & 0.07 & 0.08 & O. II & 0.17 & 0.15 & 0.15 \\
\hline $2 . I_{5} \ldots \ldots \ldots \ldots$ & 0.08 & 0.10 & $0.2 \mathrm{I}$ & 0.37 & 0.29 & 0.30 \\
\hline $2.30 \ldots \ldots \ldots$ & 0.08 & 0.13 & 0.23 & $0.4^{2}$ & $0.2 \mathrm{I}$ & 0.33 \\
\hline $2.45 \ldots \ldots \ldots \ldots$ & 0.08 & 0.13 & 0.27 & 0.48 & 0.25 & 0.43 \\
\hline $3.00 \ldots \ldots \ldots$ & 0.08 & 0.10 & 0.25 & 0.50 & 0.23 & 0.48 \\
\hline $3.15 \ldots \ldots \ldots \ldots$ & 0.15 & $0.1 \mathrm{I}$ & 0.28 & $0.5^{6}$ & 0.24 & 0.54 \\
\hline $3 \cdot 30 \ldots \ldots \ldots$ & 0.15 & 0.08 & 0.30 & 0.65 & 0.26 & 0.58 \\
\hline $3.45 \ldots \ldots \ldots \ldots$ & 0.18 & 0.09 & 0.35 & 0.70 & 0.29 & 0.63 \\
\hline $4.00 \ldots \ldots \ldots$ & 0.20 & 0.08 & 0.34 & 0.79 & $0.3 \mathrm{I}$ & 0.69 \\
\hline $4.15 \ldots \ldots \ldots$ & 0.20 & 0.08 & 0.37 & 0.87 & 0.34 & 0.78 \\
\hline $4 \cdot 30 \ldots \ldots \ldots$ & 0.23 & 0.09 & 0.38 & 0.88 & 0.35 & 0.78 \\
\hline $4.45 \ldots \ldots \ldots$ & 0.28 & 0.18 & 0.43 & 0.98 & 0.40 & 0.93 \\
\hline $\begin{array}{r}\text { June } 22 \\
8.00 \ldots \ldots\end{array}$ & 0.53 & 0.28 & 0.58 & I. 24 & 0.63 & I. 28 \\
\hline
\end{tabular}

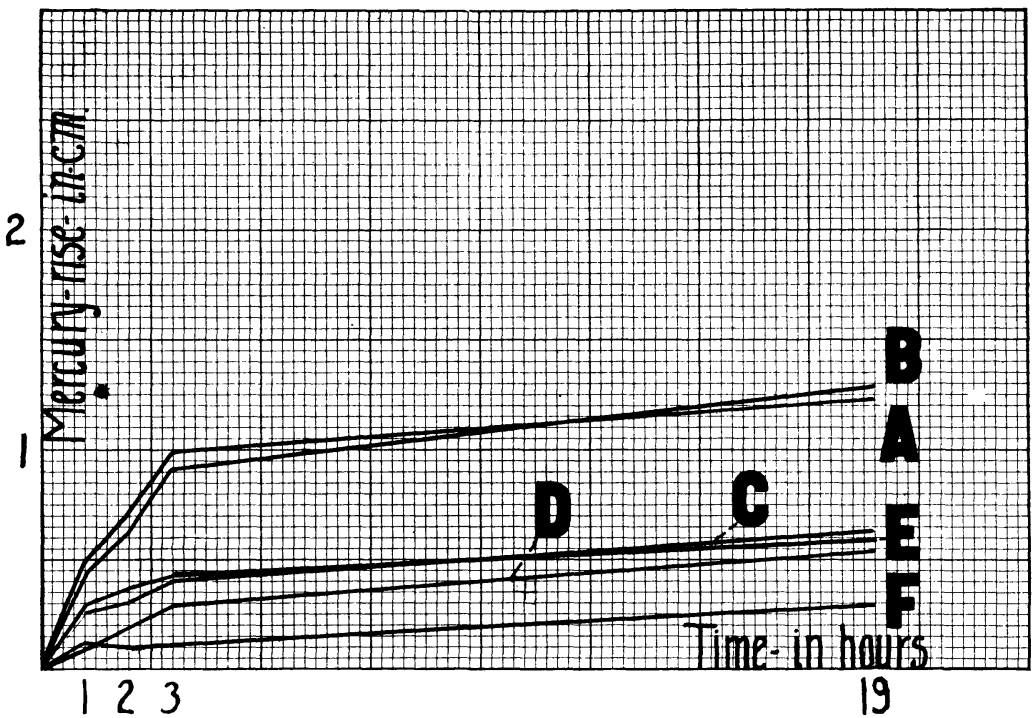

FIG. 5.- Effect of $\mathrm{MnCl}_{2}$ and $\mathrm{K}_{2} \mathrm{SO}_{4}$ on the oxidation of pyrogallol on precipitated oxidase from both healthy and diseased bark: A (diseased), $\mathrm{K}_{2} \mathrm{SO}_{4}+$ bark and pyrogallol; $\mathrm{B}$ (diseased), control (bark and pyrogallol); $\mathrm{C}$ (diseased), $\mathrm{MnCl}_{2}+$ bark and pyrogallol; D (healthy), $\mathrm{K}_{2} \mathrm{SO}_{4}+$ bark and pyrogallol; $\mathrm{E}$ (healthy), control (bark and pyrogallol); $\mathrm{F}$ (healthy), $\mathrm{MCnl}_{2}+$ bark and pyrogallol.

This content downloaded from 129.105.215.146 on September 09, 2016 21:36:14 PM All use subject to University of Chicago Press Terms and Conditions (http://www.journals.uchicago.edu/t-and-c 


\begin{tabular}{|c|c|c|c|}
\hline \multirow{3}{*}{ 品 } & \multirow{2}{*}{ 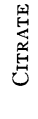 } & 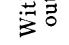 & $:$ \\
\hline & & 言 & 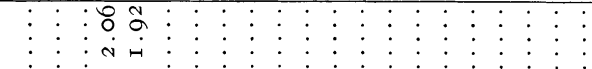 \\
\hline & \multirow{2}{*}{ 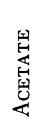 } & 常苛 & 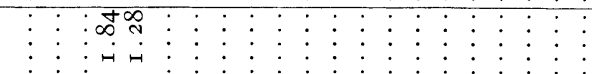 \\
\hline \multirow{3}{*}{ 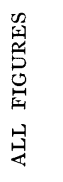 } & & 要 & $\vdots \vdots \vdots \vdots$ भी \\
\hline & & 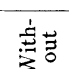 & $\vdots \vdots \vdots \vdots$ : \\
\hline & & $\mp$ & o: \\
\hline \multirow{2}{*}{$\underset{\varpi}{\stackrel{4}{a}}$} & & 3 & $\therefore$ \\
\hline & \multirow{3}{*}{ 身 } & $\underbrace{\dot{s}}_{1}$ & 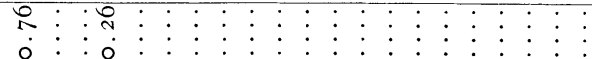 \\
\hline \multirow{2}{*}{ 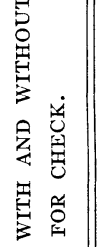 } & & 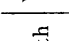 & $\widehat{N}:: \unlhd:$ \\
\hline & & 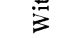 & $\dot{\sim}:: \dot{\sim}:$ \\
\hline \multirow{3}{*}{ 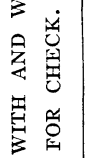 } & \multirow{3}{*}{ 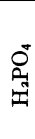 } & 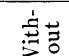 & 品 $\vdots \vdots \vdots \vdots \vdots \vdots$ \\
\hline & & & $6+2$. \\
\hline & & 焉 & 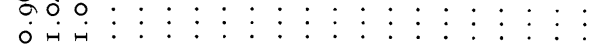 \\
\hline \multirow{4}{*}{ 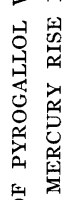 } & \multirow{3}{*}{$\stackrel{0}{\AA}$} & 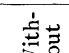 & 品 : $\vdots \vdots \vdots \vdots \vdots \vdots \vdots$ \\
\hline & & & $7: \vdots: \vdots: \vdots:$ \\
\hline & & $\underline{E}$ & $\stackrel{\infty}{\infty} \vdots \vdots \vdots \vdots \vdots \vdots$ \\
\hline & \multirow{3}{*}{ @ } & $\sum_{:=0}^{\prime}$ & 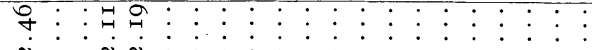 \\
\hline \multirow{4}{*}{ 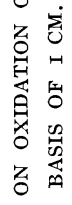 } & & & 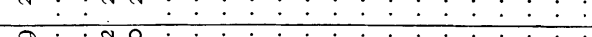 \\
\hline & & $\stackrel{5}{3}$ & 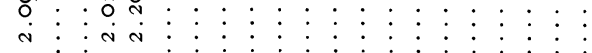 \\
\hline & \multirow{3}{*}{$0^{\infty}$} & 至芦 & 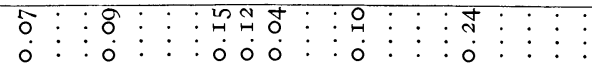 \\
\hline & & 5 & g: : \\
\hline \multirow{2}{*}{ 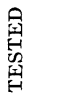 } & & 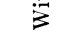 & o: : : : : : o \\
\hline & \multirow{2}{*}{ O̊ } & 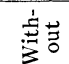 & 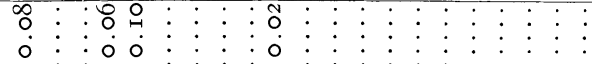 \\
\hline 皇 & & 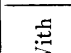 & 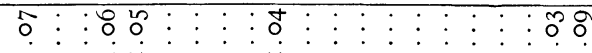 \\
\hline 본 & \multirow{4}{*}{$\vec{u}$} & & 000 in: $0: 1$ \\
\hline 安 & & 专: & o: : : $00000:$ \\
\hline 寻 & & & $0:: 00000: 0::: 0_{0}: 0::::$ \\
\hline \multirow{3}{*}{ 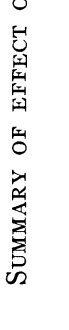 } & & 焉 & 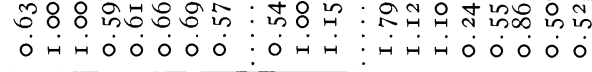 \\
\hline & & 噌 & 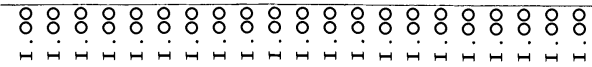 \\
\hline & & 蒫 & 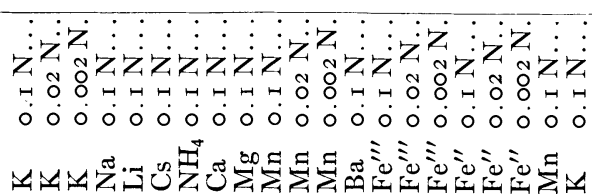 \\
\hline
\end{tabular}

This content downloaded from 129.105.215.146 on September 09, 2016 21:36:14 PM All use subject to University of Chicago Press Terms and Conditions (http://www.journals.uchicago.edu/t-and-c 


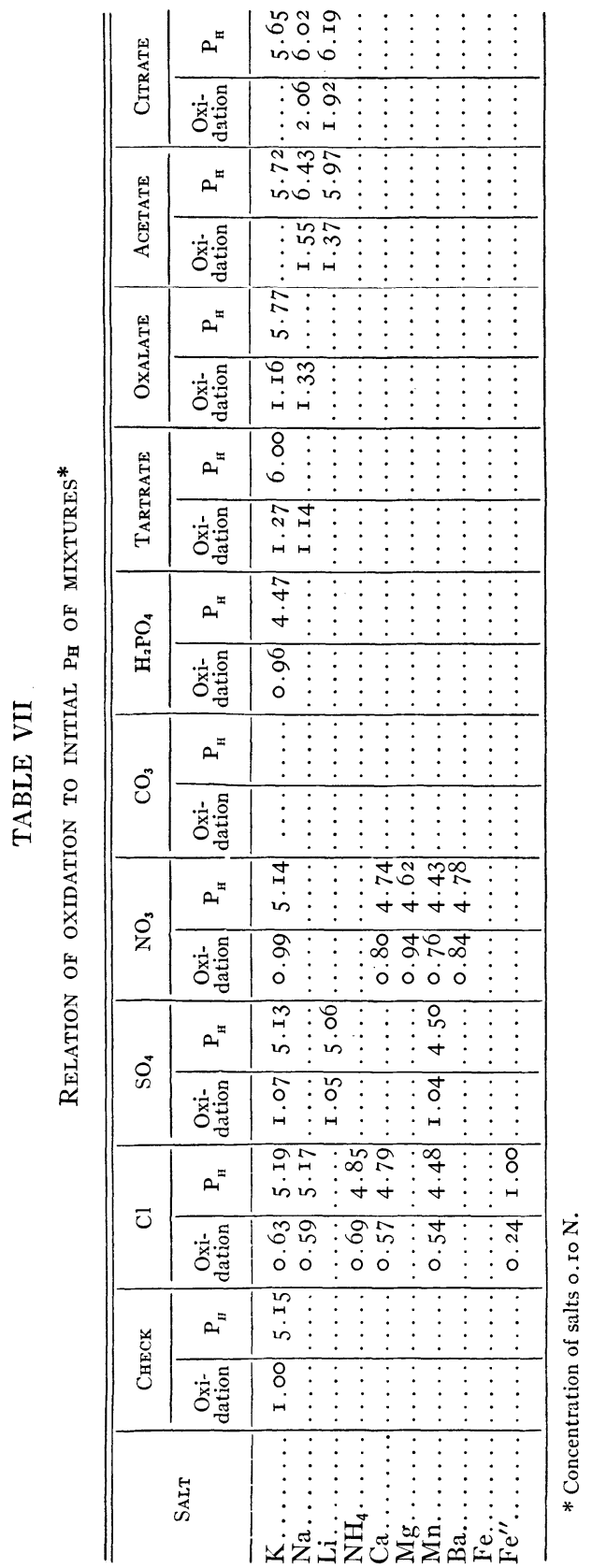

This content downloaded from 129.105.215.146 on September 09, 2016 21:36:14 PM All use subject to University of Chicago Press Terms and Conditions (http://www.journals.uchicago.edu/t-and-c 
This view is substantiated by the fact that when the concentration of ferrous chloride is reduced, oxygen absorption is reduced proportionally (table VI). If we subtract $\mathrm{I} .45 \mathrm{~cm}$. (mercury rise for pyrogallol and ferrous chloride) from $\mathrm{I} .79 \mathrm{~cm}$. (mercury rise for bark, pyrogallol, and ferrous chloride), we have $0.34 \mathrm{~cm}$. for the oxidase activity of the bark in the presence of the ferrous chloride as compared with $1.00 \mathrm{~cm}$. for the oxidase activity of bark and pyrogallol in the absence of ferrous chloride. Apparently ferrous chloride retards oxidase activity just as the other chlorides do, and the increased absorption of oxygen in the presence of ferrous chloride is due to the action of ferrous chloride itself in absorbing oxygen. Oxidation is increased by $0.002 \mathrm{~N}$ manganese chloride. This is in accord with the results of BERTRAND ( $\mathbf{5}^{\prime}$ and others. In a concentration of $\mathrm{O} . \mathrm{I} \mathrm{N}$ it inhibits oxidation just as do the other chlorides.

The use of precipitated oxidase shows that chlorides have a depressing effect on oxidation, even under conditions which eliminate many of the substances present in the bark powder. No investigation has been made of the effect of these substances on the reaction, but they probably complicate it.

The results with the chlorides are in accord with the work of EwART, who found that dilute solutions of potassium chloride and sodium chloride prevent the browning of slices of apples. EwART's further conclusion, however, that the chlorides act as sensitizers to oxidation, or ALSBERG's idea that chlorine plays an important part in the bluing of guaiacum by the chlorides of metals, are scarcely borne out by our observations that chlorides in general depress oxidase activity. It should be noted, however, that the results of those investigators were based upon color reactions, while ours were based upon oxygen absorption.

It is interesting to note that the chlorides which retard the combustion of tobacco at high temperatures have a similar effect in depressing oxidase activity. KRAYBILL (2I) has suggested that the chlorides may have a negative catalytic action in the case of the combustion of tobacco. It would be interesting to know how the chlorides affect other oxidation processes. 
The depressing effect of chlorides on oxidase activity is in contrast with their action on other enzymatic processes. Thus Nasse (25), Kübel (22), Cole (I0), Wohlgemuth (3 I), Lisbonne (23), Hawkins (18), and others have found that chlorides increase the diastatic power of various preparations of diastase. NAsse, however, found that under certain conditions sodium chloride retarded diastatic activity, and later HAwnins showed that sodium chloride and potassium chloride in certain dilute concentrations $(\mathrm{M} / \mathrm{I} 28-\mathrm{M} / 5 \mathrm{I} 2)$ retard diastatic activity. It would have been better if the effect of the chlorides upon oxidase activity had been determined in a greater number of concentrations, and it will be well in the future to do so in studying this problem. The effect of salts upon lipase activity is also of interest in this connection. LOEvENhart and Peirce (24), Gerber (i4), Terroine (30), Hamsik (i6), FALK (12), and others found that the chlorides of various alkalies and alkaline earths retard lipase activity. TERroIne found that the concentration of the salts which he studied determined the nature of their influence. Buchner, BuchNer, and HAHN (7) found that the chlorides of sodium, calcium, barium, and ammonium inhibit the fermentation of cane sugar or glucose in the presence of pressed yeast.

The results presented in table VI do not show any marked difference in the behavior of the different chlorides tested. The cations, judging from the limited data available, apparently have little or no effect; or at least their chlorides all behave very much in the same manner. In this respect the alkali salts are different in their effect upon the fire-holding capacity of tobacco, for here the salts of caesium, rubidium, and potassium in general are much more favorable to combustion than the corresponding salts of sodium or lithium. A similar contrasting behavior of different cations of chlorides was noted by HARDEN (I7), who found that potassium chloride and ammonium chloride cause a definite degree of fermentation in inactivated yeast, while sodium chloride has no effect. He says: "A specific difference in relation to alcoholic fermentation exists between the ions of sodium on the one hand and of potassium and ammonium on the other hand." Schreiner and SulLivan (29) found that potassium salts retard oxidation by the roots of plants. 
The effect of the chlorides of the alkalies in retarding oxidase activity suggests a possible practical application in preventing the browning of fruits and vegetables during their preparation for canning, preserving, or drying.

The sulphates apparently increase oxidation slightly in all cases, but the readings are not sufficiently large to be of any positive significance.

The nitrates of potassium, sodium, and magnesium have no marked effect on oxidation, while the nitrates of barium, calcium, manganese, and iron (ferric) decrease it. These results are similar to the effect upon respiration as found by ZALESKI and REINHARD (33). Fernbach and Lanzenberg (13) and Kayser (20) find that nitrates increase alcoholic fermentation, but, as they point out, the effect may be to increase multiplication of the yeast cells rather than to affect enzymatic action.

In tables II and III and figs. 2 and 3 are shown the oxidation of pyrogallol by bark alone, by bark and carbonate, and by carbonate alone. From these it is seen that in the last two cases oxidation is considerably greater than that by the bark alone. It is also seen that during the first 3 hours oxidation by carbonate is greater than that by carbonate and bark, but that after the experiment has stood overnight oxidation by healthy bark and carbonate approaches that by carbonate alone, and oxidation by diseased bark and carbonate exceeds it.

The most obvious explanation of this fact, although possibly not the true one, is that oxidation by a carbonate is a strictly chemical reaction, catalyzed only by hydroxyl ions, which soon comes to a definite end, while oxidation by carbonate and bark is a reaction catalyzed by both "oxidase" and hydroxyl ions, in which the presence of the hydroxyl ions increases the effectiveness of the "oxidase," which is slow in reaching an end-point.

Table VI shows that tripotassium phosphate increases oxidation of pyrogallol very markedly, both with and without bark. Although no $\mathrm{P}_{\mathrm{H}}$ values for this mixture are available, we know the salt is alkaline in reaction, and this effect complicates the matter. With potassium dihydrogen phosphate at 0 . 10 $\mathrm{N}$ concentration a decrease is evident, and at $0.02 \mathrm{~N}$ and $0.002 \mathrm{~N}$ concentrations a 
slight increase in oxidation occurs. The higher hydrogen ion concentration is probably the cause of the slight depression in oxidation of the 0 . Io $\mathrm{N}$ strength of the salt. The slight increase in oxidation of the lower concentrations suggests that phosphates may increase oxidase activity, but the limited data are inconclusive. It is interesting to note that IwANOFF (I9) found that phosphates raise the amount of respiration in living wheat seedlings. ZALESKI and ReInhard (33) found that disodium phosphate increases the output of carbon dioxide from dried ground seeds, and that the monobasic phosphate decreases it because of the acid reaction. These authors also quote from the work of a student, Miss Schklousky, who showed that phosphates increase the action of peroxidases, and from work of another student, Miss RosenberG, who showed that phosphates stimulate the catalase activity of different seeds.

In the case of salts of organic acids and the carbonates, all more alkaline than any of the inorganic salts (table VI), oxidation is greater at all stages of the experiment when bark is used than when it is not. Examples of this are shown in table IV. The effect of the salt is not merely additive, however, either here or in the case of the carbonates, as is shown by the following:

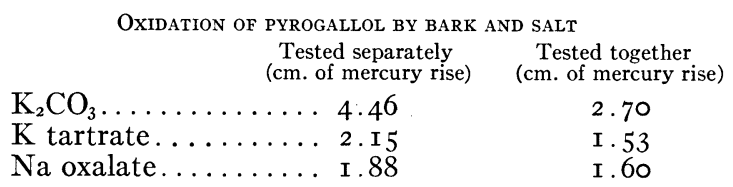

Evidently when bark and salt are combined, there is some factor at work which brings about a slower rate of oxidation than might be expected. What this factor may be we have no means of knowing as yet. Possibly it is the partial neutralization of the hydroxyl ions of the salt by the acid of the bark.

The question why salts vary so widely in the effect they have on oxidation is not easily answered. If we consider only the results with o. I $\mathrm{N}$ solutions, it seems clear, in the case of the carbonates, potassium dihydrogen phosphate, and the salts of organic acids here reported, that increased oxidation in their presence is due to the excess of hydroxyl ions they furnish; that is, by the 
reaction $\left(\mathrm{P}_{\mathrm{H}}\right)$ their solutions establish when mixed with bark and pyrogallol (table VII). The reaction established by the chlorides, however, can hardly be responsible for the decrease in oxidation they bring about, since sulphates, giving about the same reaction, cause a small increase in oxidation. For example, a mixture of potassium chloride, bark, and pyrogallol has a $\mathrm{P}_{\mathrm{H}}$ of 5 . I9 and gives only 63 per cent as much oxidation as the control. A similar mixture containing potassium sulphate has a $\mathrm{P}_{\mathrm{H}}$ of 5 . I3 and gives 7 per cent more oxidation than the control. The corresponding figures for manganese are: manganese chloride mixture, $\mathrm{P}_{\mathrm{H}}=4 \cdot 50$, oxidation $=104$ per cent of the control.

The situation for nitrates shows several irregularities. Potassium nitrate giving a $P_{\text {н }}$ of 5 . I4 has practically no effect on oxidation. Magnesium nitrate is also without effect, but gives a $P_{\text {н }}$ of 4.62. The nitrates of calcium, barium, and manganese inhibit oxidation, but manganese gives a lower $\mathrm{P}_{\text {н }}$ and the other two a higher one than that given by magnesium nitrate.

The results presented justify the conclusion that when o.I $\mathrm{N}$ solutions of the salts are used, other ions than hydrogen and hydroxyl play an important part in controlling oxidation. When hydrogen or hydroxyl ions are neutralized in making oxidase activity determinations, therefore, it is important to take into consideration the possible effect of the salts formed thereby. This must be considered as merely preliminary to the real investigations of the relation of specific ions to the oxidation processes in plants and animals. The effect of iron and manganese salts has long been known, but more work is necessary, both with these and with the more commonly occurring chlorides, sulphates, and nitrates of other cations.

\section{Summary}

I. One-tenth normal solutions of all of the chlorides tested (potassium, sodium, lithium, caesium, ammonium, calcium, manganese, ferric) decreased oxidation of pyrogallol by apple bark powder.

2. Oxidation was increased very slightly by $\circ$. Io $\mathrm{N}$ solutions of all the sulphates tested. 
3. Potassium, sodium, and magnesium nitrates ( $0.1 \circ \mathrm{N}$ ) had practically no effect on oxidation, while nitrates of calcium, barium, manganese, and iron (ferric) decreased it.

4. Potassium chloride $(0.02 \mathrm{~N}$ and $0.002 \mathrm{~N})$ had no effect on oxidation, while manganese chloride in these concentrations increased it.

5. Tartrates, oxalates, citrates, acetates, and carbonates increased oxidation. Marked increase in oxidation in these cases seems to be due, in part at least, to the low acidity of the mixtures of bark, pyrogallol, and salt.

6. Marked decrease in oxidation is not necessarily accompanied by high acidity of the mixtures.

7. Ions other than the hydrogen and hydroxyl may be important in regulating oxidase activity.

8. In neutralizing hydrogen or hydroxyl ions, it is important to take into consideration, in the study of oxidase activity, the possible effect of the salts formed thereby.

9. The chlorides which retard the combustion of tobacco at high temperatures also retard the oxidase action at low temperatures.

Io. The effect of the alkali chlorides upon oxidase activity suggests a practical application in preventing the browning of fruits and vegetables during their preparation for canning, preserving, or drying.

The authors wish to express their appreciation to Dr. WM. CROCKer for many helpful suggestions.

Bureau of Plant Industry

Washington, D.C.

\section{LITERATURE CITED}

I. Abderhalden, Emil, and Guggenheim, Markus, Versuche über die Wirkung der Tyrosinase aus Russula delica auf Tyrosin, tyrosinhaltige Polypeptide und einige andere Verbindungen unter verschiedenen Bedingungen. Zeit. Physiol. Chem. 54:33I-353. I907-1908.

2. Alsberg, Carl L., Beiträge zur Kenntnis der Guajak-Reaktion. Arch. Exp. Path. und Pharm. Festschrift. Schmiedeberg, pp. 39-53. 1908. 
3. Aso, K., On oxidizing enzymes in the vegetable body. Bull. Coll. Agric. Imp. Univ. Tokyo 5:207-235. I902.

4. BACH, A., Zur Theorie der Oxydasewirkung. II. Einflusz der Metallsalze auf die weitere Umwandlung der Produkte der Oxydasewirkung. Ber. Deutsch. Chem. Gesells. 43:366-370. I9I0.

5. Bertrand, G., Sur l'action oxydante des sels manganeux et sur la constitution chimique des oxydases. Compt. Rend. r24: I355-I358. I897.

6. - - Sur l'intervention du manganese dans les oxydations provoquèes par la laccase. Bull. Soc. Chim. I7:619-624. I897.

7. Buchner, Edward, Buchner Hans, and Hahn, Martin, Die Zymasegärung. Munchen und Berlin. I903.

8. Bunzell, H. H., A simplified and inexpensive oxidase apparatus. Jour. Biol. Chem. I7:409-4II. I9I4.

9. - - The relationship existing between the oxidase activity of plant juices and their hydrogen ion concentrations, with a note on the cause of oxidase activity in plant tissues. Jour. Biol. Chem. 28:3I5-333. I9I6.

ro. Cole, S. W., Contributions to our knowledge of the action of enzymes. I. The influence of electrolytes on the action of amylolytic ferments. Jour. Physiol. 30:202-220. 1903.

II. EwART, A. J., A comparative study of oxidation by catalysts of organic and inorganic origin. Proc. Roy. Soc. London B 88:284-320. I9I4.

12. FALK, I. S., The influence of certain salts on enzyme action. Jour. Biol. Chem. 36:229-247. I9I8.

13. Fernbach, A., and Lanzenberg, A., De l'action des nitrates dans la fermentation alcoolique. Compt. Rend. I5I:726-729. I9Io.

I4. Gerber, C., La lipase des latex, comparaison avec celle des graines. VI. Action des sels neutres, des elements halogenes et de l'eau oxygenée sur la saponification du jaune d'oeuf par la lipsae du latex d'Euphorbia Characias. Compt. Rend. Soc. Biol. 76:136-I4I. I9I4.

15. Gessard, M. C., Sur la tyrosinase. Compt. Rend. r30:I327-1330. I900.

I6. Hamsik, A., Zur Kenntnis der Pankreaslipase. Zeit. Physiol. Chem. 7I:238-25I. I9II.

I7. HARDEN, Arthur, The condition of activation of washed zymin and the specific function of certain cations in alcoholic fermentation. Biochem. Jour. Ir:64-70. I9I 7 .

18. Hawkins, LoN A., The effect of certain chlorides singly and combined in pairs on the activity of malt diastase. Bot. GAZ. 55:265-285. I9I3.

19. IwANOFF, L., Zur Frage nach der Oxydation der Gärungsprodukte des Zymins beim Atmungsprozess. Biochem. Zeit. 29:347-349. I910.

20. Kayser, M. E., Influence des nitrates sur les ferments alcooliques. Compt. Rend. I5I:8I6-8I7. I9Io.

2r. Kraybill, HenRy R., Effect of some alkali salts upon fire-holding capacity of tobacco. Bот. Gaz. 64:42-56. I9I7. 
22. KÜBEL, F., Über die Einwirkung verschiedener chemischer Stoffe auf die Thätigkeit des Mundspeichels. Archiv. für die gesammte Physiologie 76:276-305. 1899.

23. Lisbonne, Marcel, Influence des chlorures et des phosphates sur la saccharification de l'amidon demineralise par les amylases salivaire et pancreatique. Compt. Rend. Soc. Biol. 70:207-209. I9II.

24. Loevennart, A. S., and Peirce, G., The inhibiting effect of sodium fluoride on the action of lipase. Jour. Biol. Chem. 2:397-4I3. I907.

25. Nasse; Otтo, Untersuchungen über die ungenformten Fermente. Pflügers Archiv. II:I38-I66. I875.

26. Porodko, T., Zur Kenntnis der pflanzlichen Oxydasen. Beih. Bot. Centralbl. I6: I-IO. I904.

27. REED, G. B., The relation of oxidase reactions to changes in hydrogen ion concentration. Jour. Biol. Chem. 27:299-302. I9I6.

28. Rose, D. H., Blister canker of apple trees: a physiological and chemical study. Bot. Gaz. 67:105-146. I919.

29. Schreiner, O., and Sullivan, M. X.; Concurrent oxidation and reduction by roots. Bот. Gaz. 5I:273-285. I9I I.

30. Terroine, E. F., Zur Kenntnis der Fettspaltung durch Pankreassaft. Biochem. Zeit. 23:404-462. I9IO.

3I. Wohlgemuth, J., Untersuchunger über die Diastasen. I. Die tierischen Diastasen. Biochem. Zeitschr. 9: 10-43. 1908.

32. Wolff, M. J., Sur quelques proprietes nouvelles des oxydases de Russula delica. Compt. Rend. 148: 500-502. I909.

33. Zaleski, W., and Reinhard, A., Zur Frage der Wirkung der Salze auf die atmung der Pflanzen und auf die Atmungsenzyme. Biochem. Zeitschr. 27:450-473. 1910. 